\title{
Path-wise versus kinetic modeling for equilibrating non-Langevin jump-type processes
}

\author{
Mariusz Żaba, Piotr Garbaczewski and Vladimir Stephanovich \\ Institute of Physics, University of Opole, 45-052 Opole, Poland
}

(Dated: October 29, 2018)

\begin{abstract}
We discuss two independent methods of solution of a master equation whose biased jump transition rates account for long jumps of Lévy-stable type and admit a Boltzmannian (thermal) equilibrium to arise in the large time asymptotics of a probability density function $\rho(x, t)$. Our main goal is to demonstrate a compatibility of a direct solution method (an explicit, albeit numerically assisted, integration of the master equation) with an indirect path-wise procedure, recently proposed in [Physica A 392, 3485, (2013)] as a valid tool for a dynamical analysis of non-Langevin jump-type processes. The path-wise method heavily relies on an accumulation of large sample path data, that are generated by means of a properly tailored Gillespie's algorithm. Their statistical analysis in turn allows to infer the dynamics of $\rho(x, t)$. However, no consistency check has been completed so far to demonstrate that both methods are fully compatible and indeed provide a solution of the same dynamical problem. Presently we remove this gap, with a focus on potential deficiencies (various cutoffs, including those upon the jump size) of approximations involved in simulation routines and solutions protocols.
\end{abstract}

\section{INTRODUCTION.}

Investigating random dynamics is nowadays an interesting interdisciplinary research field, particularly because the same patterns of dynamical behavior can be identified and traced over in different random systems. Here the term "different" pertains both to scientific disciplines where an impact of randomness is to be analyzed and to various facets of randomness itself (diffusion versus jump process issue is here a drastic oversimplification). Concerning disciplines involved, those range from economics [1] and theory of networks [2] to biology and physics [2 4].

Quite varied randomness manifestations were either explicitly identified or theoretically and numerically tested in a a large number of physical systems. Among them we indicate glassy and porous media, nano-systems like e.g. nanoferroics (nano-structured substances with magnetic, electric and/or combined long-range orders, [5]). There, stochastic features need to be accounted for on the mesoscopic nanometer-sized level of basic (coarse-grained) constituents. The randomness (noise) input cannot be neglected if observable physical properties of the system are quantified and next confronted with experimental data.

In random systems, standard analytical methods based on presuming detailed microscopic models of stochastic dynamics (typically Langevin-based), [6], arise as an outcome of a number of simplifying steps. Therefore a potentially complete information about the system is lost, as being severely limited by the condition of analytical tractability.

If we wish to relax the tractability assumption, then to get reliable results one typically needs to turn over to direct numerical simulations (like the Monte-Carlo method, [7]). An obvious advantage of a numerical assistance is that a statistical analysis of accumulated sample paths data allow to test a congruence between theory and experiment for any random system under consideration. Moreover, various path ensemble mean values can be confronted with the single trajectory statistical features, like e.g. in connection with an ergodicity issue.

Many problems of stochastic dynamics can be analyzed by means of the "mean-field" smoothing out of microscopic quantities (random variable in the Langevin equation) and passing to macroscopic ones. That amounts to deducing a suitable differential or integro-differential equation for the probability density function (pdf) $\rho(\mathbf{r}, t)($ or $\rho(x, t)$ in one spatial dimension) whose numerical solution is seemingly easier and typically can be handled on a standard personal computer.

The present paper departs from a specific class of jump-type noises and stochastic processes whose prototype pdfs determine instantaneous spatial displacements (jumps) with a definite predominance of large ones (heavy-tailed pads and measures appropriate for symmetric Lévy flights), [4].

In the presence of conservative forces, effects of Lévy noise and its response to external potentials are normally quantified in terms of the appropriate Langevin equation, 8, 9]. However, it is well known (c.f. the Eliazar-Klafter no-go statement, [10]) that the pertinent random motion can never asymptotically set down at thermal equilibrium pdf of the Boltzmann form.

On the other hand, we have a clear physical motivation for our present research. A large class of non-Langevin Lévy-type stochastic processes has been identified, [11 13], that admit thermal equilibria in the canonical (Boltzmann) form, but theirs sample paths cannot be deduced wthin he Langevin picture. These processes generically refer to the random dynamics in inhomogeneous media, as openly stated in Ref. [12].

In this case, the main problem previously addressed in Ref. [14] was to infer a direct non-Langevin path-wise description of random motion, on the sole basis of the pre-defined master equation. The task has been accomplished 
by means of a properly tailored Gillespie's algorithm. Sample paths have been found to show up a "qualitative typicality", properly reflecting the imbalance (e.g. potential predominance of large versus small jumps), for different values of the stability index $\mu \in(0,2)$ in the related Lévy measure.

The main question, posed (and answered) in the present work, is whether the pdf $\rho(x, t)$ evolution, statistically inferred from large sample path data (the latter obtained by means of direct numerical simulations) is equivalent to that obtained from a direct numerical solution of the corresponding master equation. Once the answer is set to be positive, the next issue of interest is: which of those solution methods turns out to be more efficient (i.e. effectively faster). Our focus is on the former issue, while the latter (efficiency) topic will be briefly commented.

We quantify a pdf $\rho(x, t)$ evolution, that is driven by a non -Langevin jump-type process on $R$, in terms of the master equation, 14 16]:

$$
\partial_{t} \rho(x, t)=\int\left[w_{\phi}(x \mid y) \rho(y, t)-w_{\phi}(y \mid x) \rho(x, t)\right] d y
$$

where an integral is interpreted in terms of its Cauchy principal value and

$$
\begin{aligned}
w_{\phi}(x \mid y) & =C_{\mu} \frac{\exp [(\Phi(y)-\Phi(x)) / 2]}{|x-y|^{1+\mu}} \\
C_{\mu} & =\frac{\Gamma(1+\mu) \sin (\pi \mu / 2)}{\pi}
\end{aligned}
$$

is the jump transition rate from $y$ to $x$. We assume to have given a priori a "potential landscape" represented by a (Newtonian potential) function $\Phi(x), x \in R$ and thence an asymptotic invariant (Boltzmannian) pdf $\rho_{*}(x) \sim$ $\exp [-\Phi(x)]$ of the random process governed by Eq. (1). A concrete Lévy noise input is identified by a stability index $\mu \in(0,2)$ of the involved Lévy measure $\nu_{\mu}(d x)=\left[C_{\mu} /|x|^{\mu}\right] d x,[18$, 19]. As a standard initial data choice for the pdf dynamics we take a narrow Gaussian (mimicking the Dirac delta, e.g. a point-like source), $\rho(x, 0)=$ $\left(2 \pi \sigma^{2}\right)^{-1 / 2} \exp \left[-x^{/} 2 \sigma^{2}\right]$ with $\sigma^{2}=10^{-3}$.

We note that $w_{\phi}(x \mid y)$ typically is a non-symmetric function of $x$ and $y$. This needs to be contrasted with any "free" symmetric L evy-stable noise (take $\Phi=0$ identically) whose transition rates are $(x, y)$ - symmetric.

As long as we are interested only in a solution $\rho(x, t)$ of the master equation, we tell about a kinetic modelling of the underlying stochastic process, even if its minute details (like e.g. the random variable and related sample paths ) are not a priori defined. On the other hand, if one can devise a path-wise picture of the underlying random dynamics, whose statistical consequence is the evolution $(1)$ of $\rho(x, t)$, we tell about an indirect path-wise method of solution of the master equation. The latter case was the subject of our recent paper [14], followed by a straightforward extension from $1 \mathrm{D}$ to $2 \mathrm{D}, 17$.

Since generically there is no Langevin representation of the random dynamics in question, our main goal in Ref. [14] has been to establish the appropriate path-wise description of the underlying jump-type process and subsequently to infer the $\rho(x, t)$ dynamics directly from the random paths statistics data. However, it is not at all obvious, albeit anticipated in Ref. [14], that the path-wise route gives rise to the same evolution of the probability distribution as that coming out from a direct integration of the master equation (1). A reliable validity test is necessary here.

To deal with a tractable dynamical problem, both the indirect path-wise procedure of Ref. [14], and the direct method will be resolved in terms of a truncated jump-type process on $R$, c.f. [20]. (In passing we note that there is no jeopardy to end up with the Gaussian pdf in the large time asymptotics, which is a property of standard jump processes [20]. Our biased transition rates definitely force the random system to set down at non-Gaussian pdfs, even with cuttoffs upon the jump size.)

To this end we impose suitable cutoffs upon the original master equation (1), hereby rewritten in a more handy form $(x-y=z)$ :

$$
\partial_{t} \rho(x, t)=\int_{\varepsilon_{1} \leq|z| \leq \varepsilon_{2}}\left[w_{\phi}(x \mid z+x) \rho(z+x, t)-w_{\phi}(z+x \mid x) \rho(x, t)\right] d z .
$$

where $\varepsilon_{1}$ and $\varepsilon_{2}$ are a priori chosen, respectively lower and upper bounds for the jump size; $\varepsilon_{1}=0.001$ and $\varepsilon_{2}=1$ have been adopted in Ref. [14].

Assuming that the time increment $\Delta t$ is small enough, we can rewrite the master equation in an approximate form

$$
\rho(x, t+\Delta t) \approx \rho(x, t)+\Delta t \int_{\varepsilon_{1} \leqslant|z| \leqslant \varepsilon_{2}}\left[w_{\phi}(x \mid z+x) \rho(z+x, t)-w_{\phi}(z+x \mid x) \rho(x, t)\right] d z .
$$


whose iteration in the finite time interval $t \in[0, T]$ is expected to give positive-valued outcomes $\Delta t \ll 1$ removes a jeopardy of negative values, after a number of iteration steps).

The numerically assisted iteration procedure needs one more cutoff. Namely, for each particular choice of an asymptotic pdf $\rho_{*}(x)$ we need to establish a fine-tuned fidelity interval $[-a, a], a>0$, within which a substantial "probability mass" is concentrated. Effectively, this fine tuning amounts to selecting $a$ so that $\rho_{*}( \pm a)$ is sufficiently close to zero so that a contribution from the complement (exterior) of $[-a, a]$ in $R$ can be justifiably disregarded. A concrete parameter $a$ selection depends on the specific pdf $\rho_{*}(x)$ under consideration.

Our numerical calculation requires a partitioning of $[-a, a]$ into small pieces and storing them in a table of $\rho(x, t)$ values at every mid-point of that partition. The partition grid can not be too fine as in latter case the standard Simpson's integration in Eq. (3) unnecessarily becomes exceedingly time consuming. The speed of simulation is inversely correlated with the number of encountered partition mid-points, i.e. the partition finesse.

Comment: To make the present paper self-contained, here we briefly recapitulate the essence of our direct pathwise modelling method of the pdf dynamics (11). We refer the reader to Ref. [14] for details. Our numerical method relies heavily on Gillespie's algorithm, devised to model the dynamics of chemical reactions. In our procedure, chemical reaction channels of the original Gillespie's algorithm have been re-interpreted as jumps from one spatial point to another, like transition channels in the spatial jump process. An obvious provision is that the set of possible chemical reaction channels is finite (and generically low), while we are interested in all admissible jumps from a chosen point of origin $x_{0}$ to any of $\left[x_{0}-\varepsilon_{2}, x_{0}-\varepsilon_{1}\right] \cup\left[x_{0}+\varepsilon_{1}, x_{0}+\varepsilon_{2}\right]$. It is clear that such jumps form an infinite continuous set. It is obvious that in the numerical algorithm we cannot admit all conceivable jump sizes. As well, the number of destination points, even if potentially enormous, must remain finite for any fixed point of origin.

Our modified version of the Gillespie's algorithm, appropriate for handling of such spatial jumps reads as follows:

(i) Set time $t=0$ and the point of origin $x=x_{0}$.

(ii) Create the set of all admissible jumps from $x_{0}$ to $x_{0}+z$ that is compatible with the transition rate $w_{\phi}\left(z+x_{0} \mid x_{0}\right)$.

(iii) Evaluate

$$
\begin{aligned}
& W_{1}\left(x_{0}\right)=\int_{-\varepsilon_{2}}^{-\varepsilon_{1}} w_{\phi}\left(z+x_{0} \mid x_{0}\right) d z \\
& W_{2}\left(x_{0}\right)=\int_{\varepsilon_{1}}^{\varepsilon_{2}} w_{\phi}\left(z+x_{0} \mid x_{0}\right) d z
\end{aligned}
$$

and $W\left(x_{0}\right)=W_{1}\left(x_{0}\right)+W_{2}\left(x_{0}\right)$.

(iv) Using a random number generator draw $p \in[0,1]$ from a uniform distribution.

(v) Using above $p$ and identities

$$
\begin{cases}\int_{-\varepsilon_{2}}^{b} w_{\phi}\left(z+x_{0} \mid x_{0}\right) d z=p W\left(x_{0}\right), & p<W_{1}\left(x_{0}\right) / W\left(x_{0}\right) ; \\ W_{1}\left(x_{0}\right)+\int_{\varepsilon_{1}}^{b} w_{\phi}\left(z+x_{0} \mid x_{0}\right) d z=p W\left(x_{0}\right), & p \geqslant W_{1}\left(x_{0}\right) / W\left(x_{0}\right),\end{cases}
$$

find $b$ corresponding to the "transition channel" $x_{0} \rightarrow b$.

(vi) Draw a new number $q \in(0,1)$ from a uniform distribution.

(vii) Reset time label $t=t+\Delta t$ where $\Delta t=-\ln q / W\left(x_{0}\right)$.

(viii) Reset $x_{0}$ to a new value $x_{0}+b$.

(ix) Return to step (ii) and repeat the procedure anew.

We point out that the item (vii) in the above is most relevant for the explanation of the ultimate efficiency of path-wise simulations. The time interval is not uniquely fixed for the whole numerical procedure and directly depends on the current state of the system. 


\section{ANALYSIS OF KINETIC AND PATH-WISE OUTCOMES FOR VARIOUS TARGET PDFS AND LÉVY DRIVERS.}

\section{A. Gaussian target (harmonic confinement)}

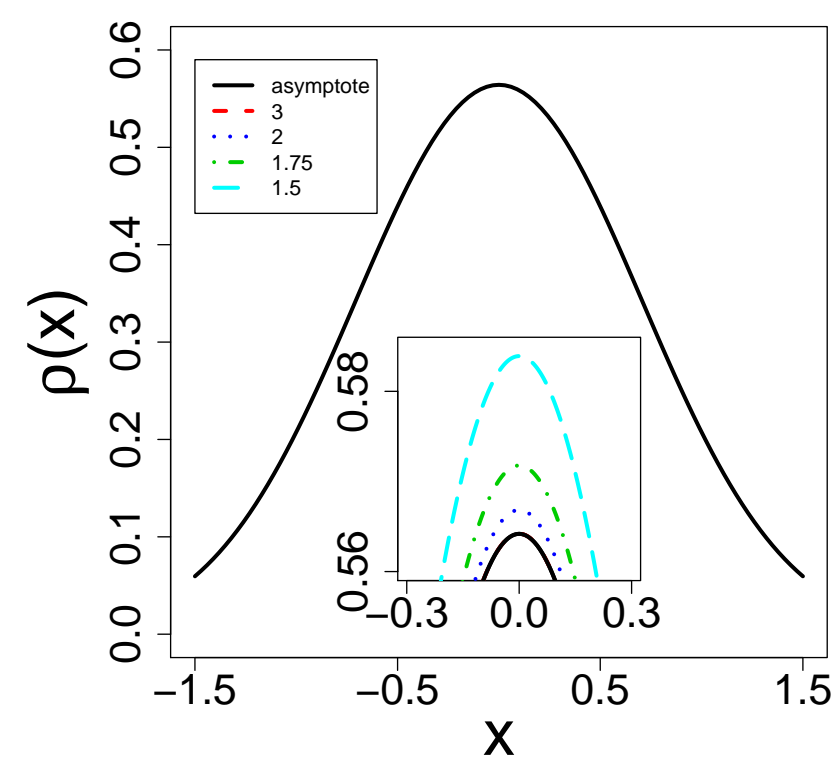

FIG. 1: Gaussian target: $x$-dependence of $\rho(x, t)$, evaluated directly from the master equation $(3)$, for $\mu=1$ and the terminal time instant $t=15$. An initial condition is $\rho(x, 0) \sim \delta(x)$, c.f. main text. The inset (note the scale change) quantifies minor differences between directly evaluated $\rho_{a}(x, t=15)$ for different $a$ values, shown in the legend. The red line $(a=3)$ in the main panel and inset cannot be distinguished from the asymptote $\rho_{*}(x)$, shown in black.

Let us consider an asymptotic invariant (target) pdf in the Gaussian form

$$
\rho_{*}(x)=\frac{1}{\sqrt{\pi}} e^{-x^{2}} .
$$

The corresponding $\mu$-family of transition rates reads

$$
w_{\phi}(z+x \mid x)=C_{\mu} \frac{e^{-z^{2} / 2-x z}}{|z|^{1+\mu}} .
$$

Fig. 1 displays a dependence of the terminal pdf $\rho(x, t=15)$ upon the choice of $a$ (e.g. the fidelity interval boundary) for the pdf $\rho_{*}(x)$. We consider the Cauchy driver $\mu=1$. With the increase of the width of the fidelity interval, the pdf $\rho(x, t)$ approaches smoothly an invariant density $\rho_{*}(x)$ of Eq. (5). In case of $a=3$ the outcome depicted in red, for all practical purposes (fapp) is indistinguishable from the Gaussian, depicted in black.

Fig. 2. displays a probability density evolution, directly computed from (3) and (4) for a fixed fidelity interval $[-3,3]$. The outcomes are compared with those inferred via an indirect path-wise method from the ensemble statistics of 75000 trajectories. Both methods clearly give consistent results.

Fig. 3 depicts the time dependence of the second moment of the involved pdf $\rho(x, t)$. The left panel in Fig. 3 displays a dependence of $\rho(x, t)$ on different fidelity interval boundary values $a$ for $\mu=1$. That is compared (path-wise method) with the statistical outcome provided by 75000 trajectories (stat indication). A numerical convergence to $<X^{2}>_{\infty}=1 / 2$ is consistent with an analytic equilibrium value of the second moment of the a priori prescribed Gaussian target $\rho_{*}(x)$. Note that the assignment of too small value $a>0$ modifies the convergence of the second 


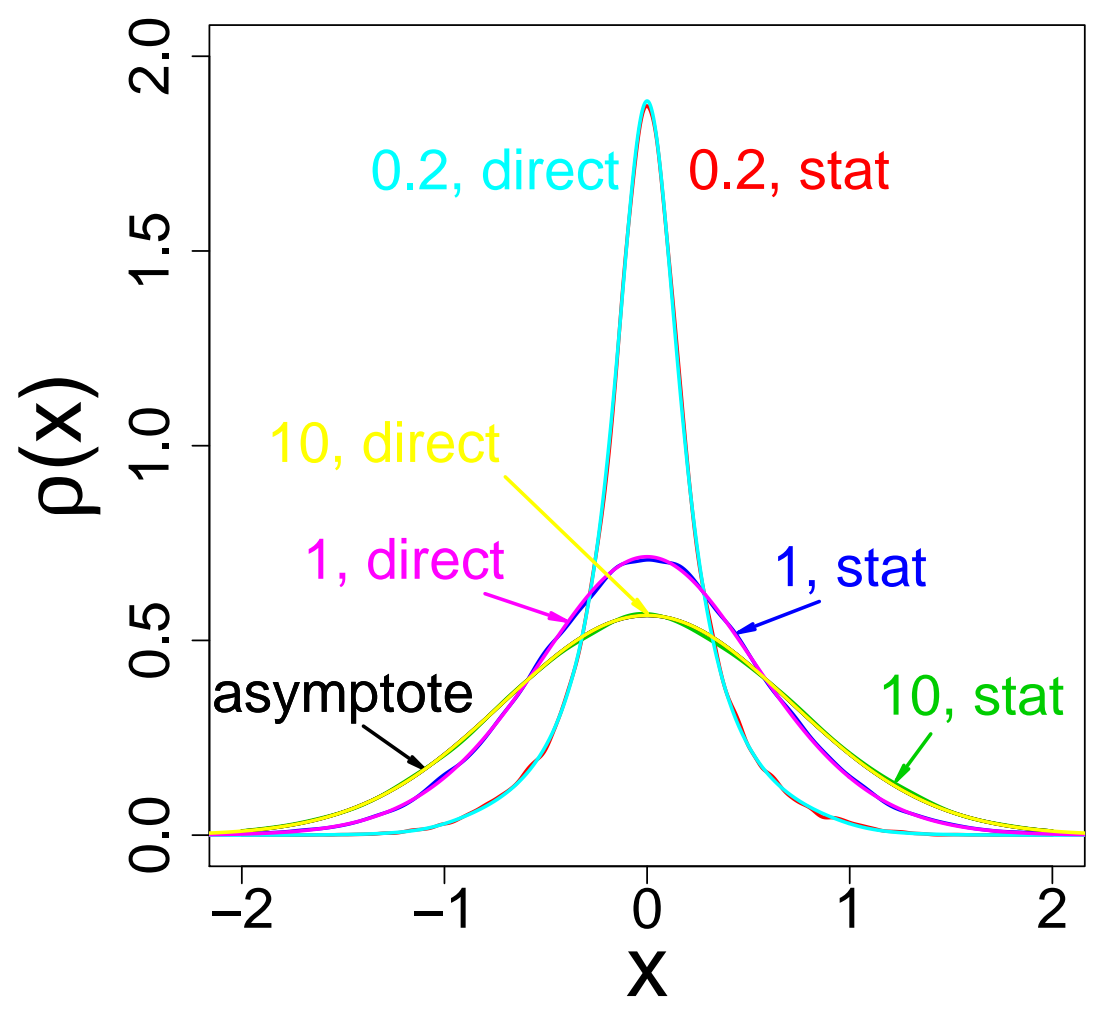

FIG. 2: Gaussian target: Comparison of the time evolution of $\rho(x, t)$ inferred from 75000 trajectories (indicated by stat) and directly from (3) (indicated by direct). We reproduce $\mu=1$ case only. The outcomes of the direct and path-wise methods are almost indistinguishable in the adopted scale. All qualitative features are preserved in case of $\mu=0.5$ and $\mu=1.5$ (not reproduced).

moment: limiting values $<1 / 2$ would necessarily appear. Indeed:

$$
<X^{2}>_{a}=\int_{-a}^{a} x^{2} \rho_{*}(x) d x= \begin{cases}0.5, & a=\infty \\ 0.49978, & a=3 \\ 0.47699, & a=2 \\ 0.44716, & a=1.75 \\ 0.39385, & a=1.5\end{cases}
$$

A middle panel in Fig. 3 displays the time dependence of the pdf $\rho(x, t)$ second moment for various $\varepsilon_{2}$ (upper bound upon the jump size), once the fidelity interval $[-3,3]$ has been adopted. It is clear that with the increase of $\varepsilon_{2}$, the asymptotic pdf (77) is approached faster.

The pertinent time rate of convergence towards an asymptotic pdf cannot be made too large, unless the fidelity interval gets much larger. One may choose $\varepsilon_{2}>3$, while keeping intact $a=3$. There is no change in the second moment temporal behavior. The right panel in Fig. 3 confirms that both direct and path-wise methods give the same evolution of the second moment, for three specific choices of Lévy drivers, namely $\mu=0.5,1,1.5$.

\section{B. Quadratic Cauchy target (logarithmic confinement)}

Let us consider an asymptotic invariant (target) pdf in form

$$
\rho_{*}(x)=\frac{2}{\pi} \frac{1}{\left(1+x^{2}\right)^{2}} .
$$



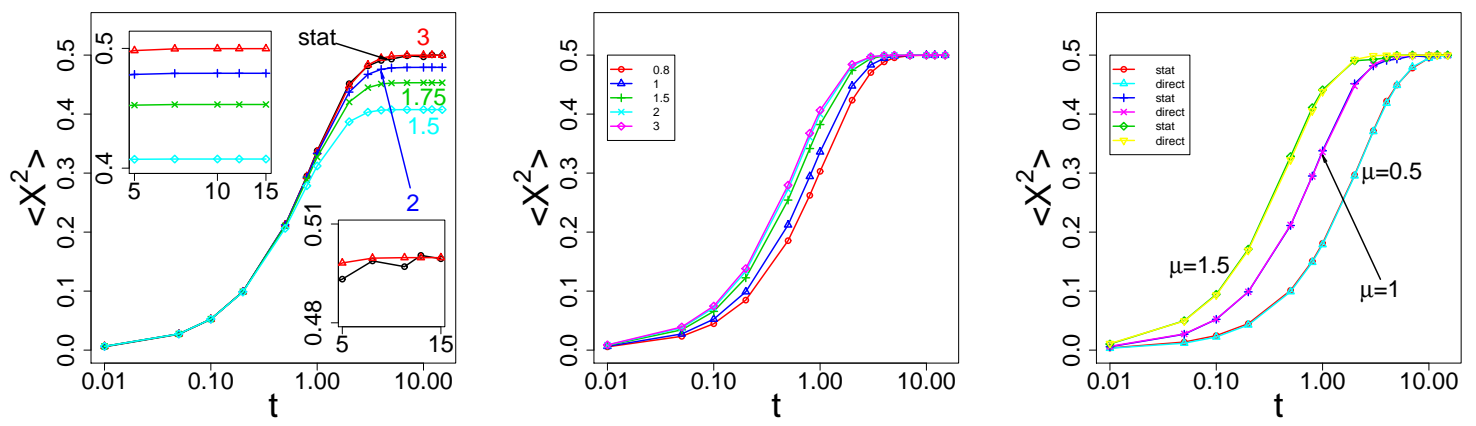

FIG. 3: Gaussian target: Time evolution of the pdf $\rho(x, t)$ second moment: dependence on $a$ for $\mu=1$ (left panel), dependence on various $\varepsilon_{2}$ for interval $[-3,3]$ and $\mu=1$ (middle panel), comparison of the two methods for various $\mu$ on $[-3,3]$ (right panel). All trajectories and probability distribution were started from a point-like source $\sim \delta(x)$.

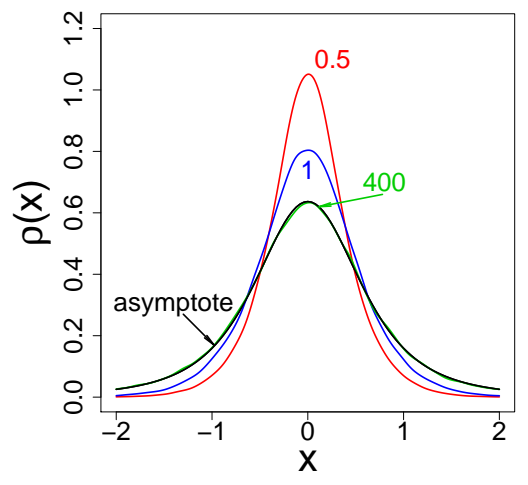

FIG. 4: Quadratic Cauchy target: time evolution of $\rho(x, t)$ evaluated directly from the master equation (3), for the fidelity interval $[-20,20]$. The $\rho(x)$ path-wise inference from 200000 trajectories (not reproduced, see however [14]) gives curves that cannot be distinguished from the former outcome.

The transition rate (2) $w_{\phi}(z+x \mid x)$ for any $\mu \in(0,2)$ takes the form

$$
w_{\phi}(z+x \mid x)=\frac{C_{\mu}}{|z|^{1+\mu}} \frac{1+x^{2}}{1+(z+x)^{2}} .
$$

Fig. 4 displays a pdf evolution that has been directly deduced from Eq. (3), for the fidelity interval $[-20,20]$. The path-wise outcome (not reproduced here, see [14]) from the ensemble statistics of 200000 trajectories gives the pdf evolution which cannot be distinguished from the former within adopted scales.

The second moment of the present $\rho_{*}(x)$, (10), equals 1 and the convergence towards this value is clearly seen in Fig. 5. Time evolution of the pdf second moment is strongly correlated with the fidelity interval boundary value $a$, c.f. the left panel and compare integration results below:

$$
<X^{2}>_{a}=\int_{-a}^{a} x^{2} \rho_{*}(x) d x= \begin{cases}1, & a=\infty \\ 0.95759, & a=30 \\ 0.93644, & a=20 \\ 0.87352, & a=10 \\ 0.75191, & a=5 \\ 0.45018, & a=2\end{cases}
$$

We note here that the fidelity interval is much broader for Cauchy type functions than that for the Gaussian one. This is related to the fact that Cauchy type functions have much slower power-law decay then Gaussians. 

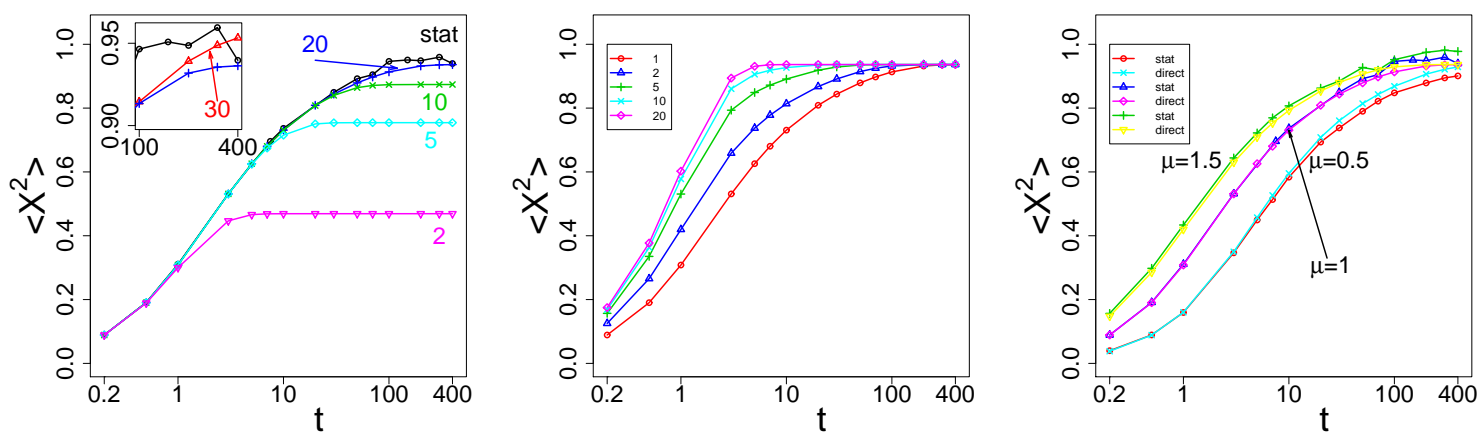

FIG. 5: Quadratic Cauchy target. Time evolution of the pdf $\rho(x, t)$ second moment. Dependence on $a$ for $\mu=1$ (left panel), dependence on various $\varepsilon_{2}$ for the fidelity interval $[-20,20]$ and $\mu=1$ (middle panel). Comparison of two solution methods for various $\mu$ and $[-20,20]$ (right panel). All trajectories and probability distribution were started point-wise $\rho(x, 0) \sim \delta(x)$.

The middle panel in Fig. 5 displays the time dependence of the pdf $\rho(x, t)$ second moment for various $\varepsilon_{2}$ and the fidelity interval $[-20,20]$. Like in the gaussian case, the target pdf (10) is achieved faster if we increase $\varepsilon_{2}$.

The direct and path-wise (indirect) methods of solution of the master equation (3) are depicted in the right panel of Fig. 3, for three specific choices of Lévy drivers, namely $\mu=0.5,1,1.5$. The agreement is good and can be easily made better if the trajectory statistics data are increased to more than 200000 trajectories and/or the fidelity interval gets increased in the direct solution method.

\section{Cauchy target: non-Langevin modeling of Boltzmannian equilibration.}
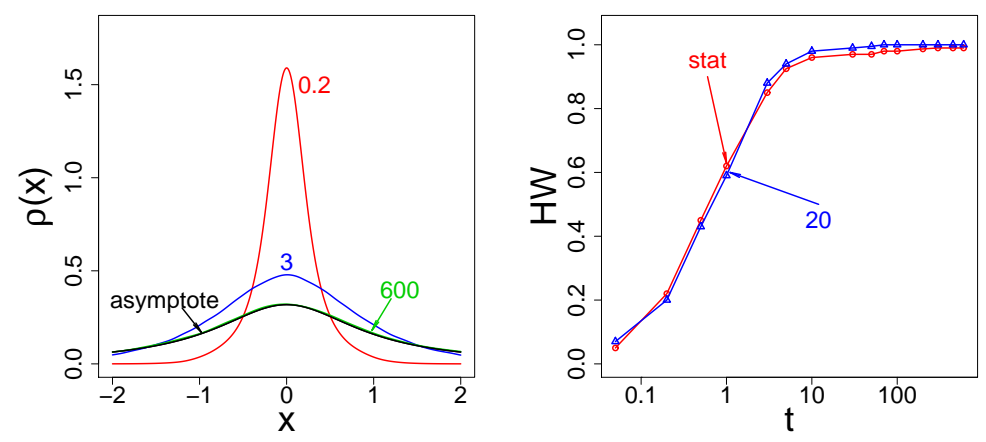

FIG. 6: Cauchy target. Comparison of the time evolution of $\rho(x, t)$ inferred from 200000 trajectories and directly from (3). They are indistinguishable on the left panel. The half-width (HW) temporal behavior is depicted in the right panel. The initial condition is point-wise $\rho(x, t=0) \sim \delta(x)$.

Now we consider an asymptotic (target) pdf to be given in the familiar Cauchy form. The dynamical scenarios that asymptotically set down at the Cauchy pdf, may serve as a useful diagnostic tool when comparing the intricacies (similarities and differences) of the Langevin and non-Langevin equilibrating dynamics, with common initial data and the common terminal pdf.

Right at this point the major difference can be seen between the non-Langevin motion scenario, for which the Bolztmannian (e.g. thermal) equilibration is possible, and the standard Langevin modeling, to which the EliazarKlafter no-go statement readily extends, see e.g. 10, 14].

The classic Cauchy density is associated both with the free Cauchy noise (probability distribution of random jumps) 
and/or the confining Ornstein-Uhlenbeck-Cauchy process [21]. We have

$$
\rho_{*}(x)=\frac{1}{\pi} \frac{1}{1+x^{2}} .
$$

In this case, the transition rate from $x$ to $x+z$ reads

$$
w_{\phi}(z+x \mid x)=\frac{C_{\mu}}{|z|^{1+\mu}} \sqrt{\frac{1+x^{2}}{1+(z+x)^{2}}} .
$$

We consider the Cauchy driver, e.g. $\mu=1$. In Fig. 6 we report the directly resolved time evolution of the corresponding pdf, its half-width (the second moment does not exist in the present case) for different time instants. The left panel in Fig. 6 displays results evaluated directly from (3), for the fidelity interval $[-20,20]$. The pathwise (indirect method) outcome inferred from 200000 trajectories is indistinguishable within the scales adopted. An approach to the asymptotic Cauchy pdf is clearly seen, together with a convergence of a half-width to its asymptotic value 1. The right panel of Fig. 6 compares the half-width of $\rho(x, t)$ temporal behavior, obtained by means of direct (indicated by 20) and path-wise (stat) methods of soplution of Eq. (3). Slight differences in the curves result from numerical approximations.

Remark: Displayed path-wise inferred curves in Fig. 6 (right panel) are hampered by certain errors. The figures have been read from a histogram of randomly sampled data. A partitioning into subintervals, resulting in the histogram shape, is a source of inaccuracies. One more inaccuracy source in the fine partition case comes from the maximum read-out imprecision. Therefore the partitioning finesse always needs an optimization. The time evolution of pdf, obtained directly from the master equation (3), depends on the fidelity interval adopted. This choice contributes to maximum and half-maximum of the curve read errors.

\section{Cauchy target: Langevin-type (OUC) equilibration.}

To make a clear distinction between the Langevin and non-Langevin modeling, we shall describe an alternative equilibration scenario, realizable as rather standard Ornstein-Uhlenbeck-Cauchy (OUC) process, where the Bolzmann thermalization is excluded by the argument of Ref. [10].

Namely, with a slighly modified Cauchy density, we can associate not only the free Cauchy noise, but also the confining OUC process. Its drift is given by $b(x)=-\nabla \Phi(x)=-\gamma x$, where we have adopted $\Phi$ as the Newtonian harmonic potential. An asymptotic invariant pdf associated with the standard fractional (Cauchy) Fokker-Planck equation

$$
\partial_{t} \rho=-\lambda|\nabla| \rho+\nabla[(\gamma x) \rho]
$$

reads:

$$
\rho_{*}(x)=\frac{\sigma}{\pi\left(\sigma^{2}+x^{2}\right)},
$$

where $\sigma=\lambda / \gamma$, c.f. Eq. (9) in Ref. [21]. In accordance with the Eliazar-Klafter no-go statement, [10], the equilibrium pdf does not admit a standard Boltzmannian form $\rho_{*}(x) \sim \exp [-\Phi(x)]$.

Since the OUC pdf (16) has no variance, in Fig. 7 we visualize the temporal evolution of $\rho(x, t)$, started from $\rho(x, t=0) \sim \delta(x)$ for two motion scenarios corresponding to the Langevin (OUC) and non-Langevin cases, provided they share a common Cauchy target pdf. In addition to the direct comparison of the shapes of $\rho(x, t)$ in above evolution scenarios, for both cases we plot the width of the OUC "bell" at its half-maximum at a number of consecutive instants of time. The latter can serve as an diagnostic tool, differentiating between those definitely inequivalent motions, see also Refs. [12, 13].

\section{OUTLOOK}

We have completed a compatibility test for two different procedures that can be employed to solve the master equation (1). By the construction we deal with non-Langevin jump-type processes, whose jump intensity is induced by a properly biased Lévy - stable noise.

In the present paper, without limiting a generality of results, we have confined our attention to the most difficult (from the convergence point of view) case of the non-Gaussian Cauchy driver and its varied forms of response to 

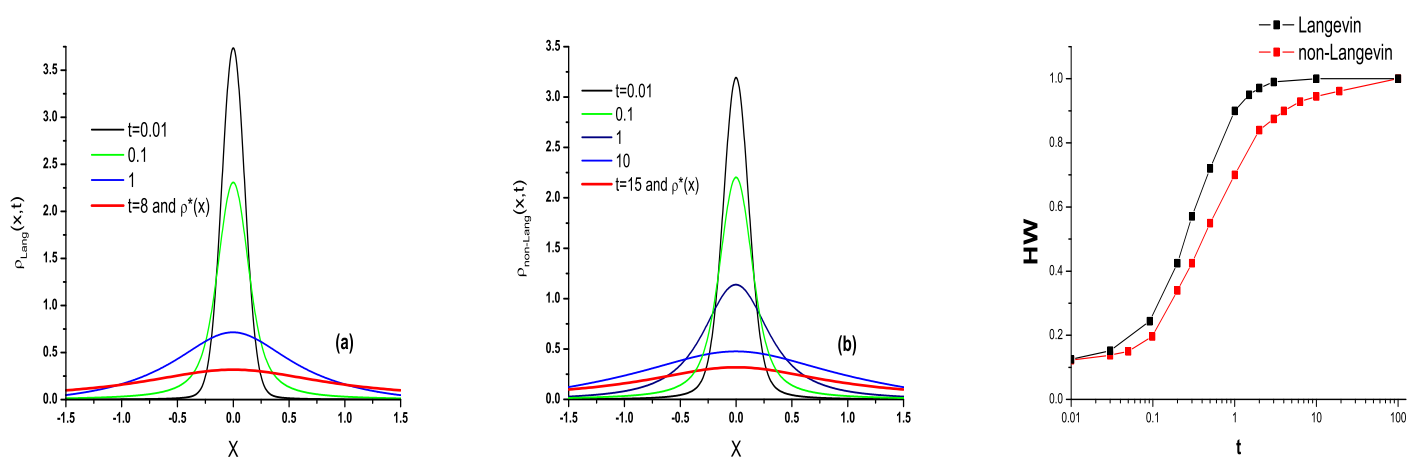

FIG. 7: Time evolution of pdf's $\rho(x, t)$ for the Cauchy-Langevin dynamics (panel (a)). The non-Langevin motion scenario is reported in panel (b). For both scenarios, the common target pdf is the Cauchy density, while the initial $t=0$ pdf is set to be a Gaussian with height 25 and half-width $\sim 10^{-3}$. The first depicted stage of evolution corresponds to $t=0.01$. Panel (c) reports a difference between the Langevin and non-Langevin patterns of dynamical behavior in terms of the half-width of the corresponding pdfs.

external potentials. In particular, for an exoplcit choice of the $\mu=1$ Cauchy driver, we have discussed a difference between the Langevin and non-Langevin dynamical patterns of behavior. Those, while being started from the same initial (Dirac delta-like) pdf data, ultimately result in the common for both asymptotic Cauchy target pdf. Albeit following inequivalent time evolution patterns of behavior.

Within the non-Langevin modeling an emergence of the Cauchy target pdf can be consistently interpreted as an asymptotic outcome of the thermal equilibration process, see e.g. also Ref [11]. The Boltzmann form of the pdf is fully compatible with the non-Langevin framework. To the contrary, the standard Langevin modeling is known to be incompatible with the Boltzmannian equilibration, c.f. [10].

We have emphasized before that a leading physical motivation an investigation of non-Langevin jump-type processes, compatible with the master equation (1), was an observation 11] that Lévy type pdfs can be receive an interpretation of Boltzmann equilibria. However, the price to be paid is that we must go beyond the tenets of a popular paradigm of the Langevin modeling of Lévy processes.

One may argue that a trui interest of the method is its application to stochastic problems of non-physical origin, like econ omics or population dynamics. A standard argument is that if those come from physics a Langevin formulation seems to be appropriate and adequate. Our point is that the Langevin modeling of Lévy, quite against the standard lore, is inadequate. More than that, the evident spatial non-locality present in the definition of noise generators as classified by the Lévy-Khintchine formula, needs a careful exploration as well, see e.g. [26].

Coming back to purely physical arguments, we note here that the non-Langevin master equation (1D) may be interpreted as a direct analog of the Boltzmann kinetic equation derived for pairwise interparticle interactions [22]. This equation has been successfully applied to describe nonequilibrium properties of different physical systems like dielectrics or superconductors. It drives a kinetics of first order phase transitions 22]. Another field, where the non-Langevin master equation (11) has been in fact used, is the theory of radio spectroscopic methods, like election paramagnetic and nuclear magnetic resonances.

The master equation of the form (11) is used as well in the description of spin-lattice relaxation in solids, liquids and gases. These processes originate from spins motion, induced by an external (probing) magnetic field. They lead to the so-called dynamic broadening of resonant lines, [23].

The very same master equation, with or without the detailed balance principle imposed to hold true, has been applied to the problem of so-called statistical mechanics of money 24]. This problem, related to the application of methods of statistical physics in economy (so-called econophysics [1]), deals with master equation (11) with properly (due to peculiarities of the specific problem under consideration) modified transition rates (2).

Namely, in Ref. [24], Eq. (11) has been solved numerically to obtain the outcome of the "thermal" equilibration of the process of the random exchange of money between several agents. The outcome turns out to be of exponential, i.e. non-Gaussian form. The results of numerical solution of the Boltzmann-type equation have been compared in Ref. 24] with direct, trajectory-wise numerical simulations, performed earlier [25]. The methods, developed in the present paper, are capable to model adequately the above class of problems.

Our present study shows clearly that the direct numerical solution of the master equation (1D) is sufficient to obtain the reliable information about the stochastic dynamics in question. Although, as a matter of principle, we may not 
even invoke the notion of random trajectories underlying the pertinent pdf dynamics, given the master equation data - we can always retrieve them (e.g. trajectories) back. The Gillespie's algorithm is a useful tool to this end, as demonstrated in Ref. [14].

We emphasize that it is sometimes useful to resort to the indirect path-wise methods, based on the above mentioned algorithm. Specifically it is the case, when the master equation of the type (1) is a priori known and one attempts to devise (model) or deduce an underlying microscopic model of random motion. This viewpoint is particularly important if the standard Langevin modeling does fail.

To compare the efficiency and computer time consumption of both direct and indirect algorithms to solve (1) is not trivial at all and cannot be settled unambiguously.

A direct integration of Eq. (1) needs some care regarding the spatial interval $[-a, a]$ and time interval (hence fixing a sufficiently small value of $\Delta t$ ) partitioning finesse. The ultimate computer time consumption depends quite sensitively on the proper balance between those two partitioning options.

The path-wise method appears to be much more computer time demanding than the direct kinetic modeling. A dynamical retrieval of a single $\rho(x, t)$, needs so many separate runs in the path-wise approach, that the computer simulation and subsequent acquisition of sufficiently large statistical data may take not merely minutes, but days or weeks. Typically the path-wise method needs a simulation and accumulation (data storage) of a large number of trajectories, typically between $10^{3}$ and $3 \cdot 10^{3}$. Even with the parallel computing on small clusters the procedure may take days, for larger values of the stability index $\mu \in(0,2)$. Especially when increasing the spatial dimensionality from $1 d$ to $2 D,[17]$, or $3 D$.

The major gain of the path-wise method is that we can adequately simulate a microscopic motion scenario that is compatible with the dynamics (1).

Remark: Since quite varied (here Langevin and non-Langevin respectively) microscopic motion scenarios can be used to implement a dynamical interpolation between any given a priori pair of initial (here narrow Gaussian) and target pdf data, we get a reason to mention and advocate a complementary viewpoint, based on the exploitation of diffusion-type processes, instead of jump-type ones. In this connection, we mention our recent [13] finding that, under suitable confinement conditions (i.e. properly tailored external potentials) and in clash with the folk lore ways of thinking in this context, the ordinary Fokker - Planck equation may generate non-Gaussian heavy-tailed pdfs, (like Cauchy or more general Lévy-stable distributions) in its long-time asymptotics. Specifically, the problem of physical relevance is, whether the three dimensional Fokker - Planck equation, in the presence of a specific external potential, can be employed to describe the non-ergodic long-time relaxation in glassy systems. This issue is presently under investigation.

[1] R.N. Mantegna and H.E. Stanley, An Introduction to Econophysics. Correlations and Complexity in Finance, Cambridge Univ. Press, Cambridge, 2000

[2] S.N. Dorogovtsev and J.F.F. Mendes, Evolution of Networks: From Biological Nets to the Internet and WWW, Oxford University Press, Oxford, 2003

[3] N.G. van Kampen, Stochastic Processes in Physics and Chemistry, North-Holland, Amsterdam, 1981

[4] Lévy flights and Related Topics in Physics,(edited by M. F. Shlesinger, G.M. Zaslavsky and U. Frisch), Lecture Notes in Physics, Springer-Verlag, Berlin, 1995; Chaos: The Interplay between Deterministic and Stochastic Behavior, (edited by P. Garbaczewski, M. Wolf and A. Weron), Springer-Verlag, Berlin, 1995

[5] M.D. Glinchuk, A.V. Ragylya and V.A. Stephanovich, Nanoferroics, Springer, Dordrecht, 2013

[6] M. Mezard, G. Parisi, M. Virasoro, Spin Glass Theory and beyond, World Scientific. Singapore, 1987

[7] K. Binder and D.W. Heermann Monte Carlo Simulation in Statistical Physics. An Introduction (4th edition). Springer, Dordrecht, 2002

[8] S. Jespersen, R. Metzler and H. C. Fogedby, Phys. Rev. E59, 2736, (1999)

[9] R. Metzler et al, Europhys. Lett. 46, 431, (1999)

[10] I. Eliazar and J. Klafter, J. Stat. Phys. 119, 165, (2005)

[11] P. Garbaczewski and V. Stephanovich, Open Systems Inf. Dyn., 17, 287, (2010)

[12] P. Garbaczewski and V. A. Stephanovich, Physica A 389, 4419, (2010)

[13] P. Garbaczewski, V. A. Stephanovich and D. Kȩdzierski, Physica A 390, 990, (2011)

[14] M. Żaba, P. Garbaczewski and V. Stephanovich, Physica A 392, 3485, (2013)

[15] D. Brockmann and T. Geisel, Phys. Rev. Lett. 90, 170601, (2003)

[16] V. V. Belik and D. Brockmann, New. J. Phys. 9, 54, (2007)

[17] M. Żaba, P. Garbaczewski, International Journal of Statistical Mechanics, vol. 2013, 738345, (2013); doi:10.1155/2013/738345

[18] K.-I. Sato, Lévy Processes and Infinitely Divisible Distributions, Cambridge University Press, Cambridge, 1999 
[19] J. Lörinczi, F. Hiroshima and V. Betz, Feyman-Kac-Type Theorems and Gibbs Measures on Path Space, Studies in Mathematics 34, Walter de Gruyter, Berlin, 2011

[20] R. N. Mantegna and H. E. Stanley, Phys. Rev. Lett. 73, 2946, (1994)

[21] P. Garbaczewski and R. Olkiewicz, J. Math. Phys. 41, 6843, (2000)

[22] E. M. Lifshitz and L. P. Pitaevskii, Physical Kinetics (Pergamon Press, New York, 1993)

[23] A. Abragam, Principles of Nuclear Magnetism, (Oxford University Press, Oxford, 2002)

[24] A.A. Drăgulescu and V.M. Yakovenko, Eur. Phys. J. B, 17, 723 (2000), see also A.A. Drăgulescu PhD Thesis, arXiv:cond-mat/0307341

[25] S. Ispolatov, P. L. Krapivsky, and S. Redner, Eur. Phys. J. B 2, 267 (1998)

[26] P. Garbaczewski and V. Stephanovich, J. Math. Phys. 54, 072103, (2013) 